\title{
Recomendaciones en el abordaje diagnóstico y terapéutico del fumador. Documento de consenso
}

\author{
M. Torrecilla García, F. Domínguez Grandal, A. Torres Lana, C. Cabezas Peña \\ Por la Sociedad Española de Medicina Familiar y Comunitaria (semFYC). \\ Grupo de Abordaje del Tabaquismo de la semFYC \\ C. A. J iménez Ruiz, M. Barrueco Ferrero, S. Solano Reina, J I. de Granda Orive \\ Por la Sociedad Española de Neumología y Cirugía Torácica (SEPAR) \\ J. L. Díaz-Maroto Muñoz, J. Alonso, M. L. Martínez, S. García \\ Por la Sociedad Española de Medicina Rural y Generalista (SEMERGEN) \\ E. de la Cruz Amorós, R. Abengozar Muela, f. Ramos Postigo, J. Ayesta \\ Por la Sociedad Española de Especialistas en Tabaquismo (SEDET)
}

\section{RESUMEN}

El tabaquismo es la principal causa de muerte evitable en los países desarrollados. Todos los profesionales sanitarios jugamos un papel clave en su control. Es fundamental realizar un correcto diagnóstico de las características de cada fumador para de esa forma ofertarle su tratamiento más adecuado. A lo largo de este documento de consenso entre las sociedades científicas que agrupan a los profesionales sanitarios más interesados en el tabaquismo, hemos definido un grupo de datos cli nicos y analíticos que deben ser considerados para establecer el conjunto mínimo de datos diagnósti cos en el fumador. Además recomendamos una ac tividad terapéutica adecuada a las características diagnósticas de cada fumador. Este conjunto míni mo de datos es la base imprescindible para esta blecer el tratamiento más adecuado a cada indivi duo con el objetivo de que el paciente progrese en el proceso de abandono del tabaco y consiga la abstinencia tabáquica en el más corto periodo de tiempo posible y con el menor coste personal. Así mismo este conjunto mínimo de datos permite ra cionalizar la intervención del profesional sanitario conforme a criterios de eficacia y eficiencia.

Palabras clave: Tabaquismo. Diagnóstico. Abandono de tabaco. Documento de consenso.
Recommendations in diagnosis and treatment of tobacco cessation. Consensus document

\begin{abstract}
Consumption of tobacco is the main cause of avoidable death in the developed countries. All the sanitary professionals play a key paper in their control. It is fundamental to carry out a correct diagnosis of each smoker's characteristics for offering him their more appropriate treatment. Along this consensus document among the scienti fic societies that contain the most interested sani tary professionals in the treatment of tobacco, it has been defined a group of clinical and analytic data that should be considered to establish the mi nimum group of data diagnoses in the smoker. We also recommend an appropriate therapeutic acti vity to each smoker's characteristic. This minimum group of data is the indispensable base to establish the most appropriate treatment to each individual with the objective of that the patient progresses in its process of tobacco cessation and stop to smoke in the shortest period of time and with the smallest personal cost. Likewise this minimum group of data allows to rationalize the intervention of the sani tary professional according to approaches of effec tiveness and efficiency.
\end{abstract}

Key words: Tobacco. Diagnosis. Tobacco cessa tion. Consensus document.

Aceptación: 24-06-02 


\section{INTRODUCCIÓN}

De acuerdo a los más recientes datos estadísticos sabemos que el $36 \%$ de la población general española, mayor de 16 años de edad es consumidora habitual de tabaco y que el $70 \%$ de los fumadores visita, al menos una vez al año, a su médico de familia $^{1,2}$. Además, conocemos que el tabaquismo representa la primera causa evitable de muerte en nuestro país y que se ha calculado que cada año mueren 56.000 españoles por enfermedades directamente relacionadas con el consumo de tabaco ${ }^{3}$. Estos datos deben alertar a todos los profesionales sanitarios para que durante su práctica asistencial desarrollen intervenciones sobre el tabaquismo. Cualquier tipo de intervención terapéutica que los médicos realicen sobre el tabaquismo de sus pacientes es eficaz, ahora bien, cuando la intervención se adecua a las características individuales de cada fumador, su eficacia y eficiencia aumentan .

Por otro lado, en el momento actual, el tabaquismo es considerado como una enfermedad adictiva crónica susceptible de recibir diferentes tipos de tratamiento $^{4-6}$. Es por ello, por lo que conviene definir parámetros clínicos y analíticos del tabaquismo que nos lleven a establecer un correcto diagnóstico del tipo de tabaquismo que un determinado fumador padece. De esta forma, conseguiremos pautar el tratamiento más adecuado para ese fumador y con mayores probabilidades de éxito.

El principal objetivo de este artículo es definir un grupo de parámetros clínicos y analíticos que deban ser obligatoriamente analizados en cada fumador, por cuanto que su conocimiento influirá directamente no sólo en nuestra actitud terapéutica ante el mismo, sino también en su pronóstico. Estableceremos, pues, a lo largo de esta revisión lo que hemos dado en llamar: Conjunto Mínimo de Datos Diagnósticos del Fumador, que deberán ser de obligada referencia en la historia clínica de todo fumador. Con posterioridad, y teniendo en cuenta los resultados obtenidos en el estudio del conjunto mínimo de datos diagnósticos, estableceremos unas recomendaciones terapéuticas.

\section{Conjunto Mínimo de Datos Diagnósticos en el Fumador (CMDF)}

Para la valoración de estos datos se tendrán en cuenta los siguientes parámetros:

\section{Cantidad de tabaco consumido}

Éste es el parámetro que se recoge con mayor frecuencia en cualquier historia clínica de un fumador. Pero no sólo es necesario hacer referencia al número de cigarrillos consumidos al día, sino también al tiempo transcurrido como fumador, lo que orienta sobre algunas características del hábito tabáquico y en consecuencia de la dificultad de cambiar su conducta adictiva. Estos dos datos quedan englobados en el concepto: número paquetes/año. Para determinar esta cifra se realiza una sencilla operación: se multiplica el número de cigarrillos consumidos al día por el fumador por el número de años que lleva consumiendo esa cantidad de tabaco, y su resultado se divide por 20. De tal manera que un fumador de 20 cigarrillos diarios durante 20 años, consumirá un total de 20 paquetes/año.

Aunque no existe un umbral de seguridad para el consumo del tabaco y tan perjudicial es consumir 20 paquetes/año como 3 , también es cierto, que en la mayor parte de los estudios realizados que analizan la relación entre el consumo del tabaco y el padecimiento de un buen número de enfermedades se observa una relación dosis/respuesta, de tal manera, que la posibilidad de desarrollar enfermedades relacionadas con el consumo del tabaco es más alta en los fumadores de un mayor número de cigarrillos diarios que en aquéllos que consumen una menor cantidad, sin que sea posible señalar un determinado umbral de seguridad ${ }^{7-9}$.

Además sabemos que existe una relación entre el número de cigarrillos consumidos al día y el número de años de fumador y la posibilidad de dejar de fumar en un determinado intento de abandono. Así, los fumadores que consumen mayor número de cigarrillos al día y aquéllos que más tiempo llevan fumando son los que más dificultades experimentan ante un intento de abandono y en consecuencia tienen unas posibilidades más bajas de dejar de fumar ${ }^{10,11}$.

Vemos cómo el conocimiento de este parámetro, número de paquetes/año, nos puede servir para valorar la posibilidad de desarrollar enfermedad o, incluso, las posibilidades de éxito o fracaso de un intento de abandono.

\section{Fase de abandono}

Prochazka y DiClemente establecieron las fases que la mayor parte de los fumadores atraviesan desde que se inician al consumo del tabaco hasta que lo abandonan ${ }^{12}$. Estos investigadores definieron cinco fases: precontemplación, contemplación, preparación, acción y mantenimiento. Los fumadores se encuentran en una $u$ otra dependiendo de su mayor o menor motivación para dejar de fumar y de su mayor o menor inclinación a realizar un serio intento de abandono. Es fundamental que ante todo fumador investiguemos la fase de abandono en la que se encuentra, pues la actitud terapéutica que tengamos con él, vendrá determinada por esta fase. 
El conocimiento de la fase de abandono nos permitirá instaurar el tratamiento más adecuado para cada fumador ${ }^{4,13}$.

\section{Motivación para el abandono del tabaco}

Estrechamente relacionado con las fases de abandono del tabaco está el grado de motivación para intentarlo. En una primera aproximación es aconsejable conocer si el fumador está dispuesto realizar un "serio" intento de abandono del tabaco y para ello basta con preguntárselo directamente y observar la actitud del mismo. Posteriormente, pero quizás fuera ya de lo que podría catalogarse de datos mínimos indispensables en una primera valoración del paciente fumador, podrían realizarse varios cuestionarios elaborados para dicho fin, de los que uno de los más conocidos y usados es el test de Richmond.

\section{Test de Fagerström}

El Test de Fagerström es el instrumento más útil, de los que disponemos en el momento actual, para medir el grado de dependencia física que los fumadores tienen por la nicotina ${ }^{14}$. Se trata de un test de seis preguntas con respuestas múltiples. Depen- diendo de la respuesta que cada fumador dé a cada una de las preguntas se obtiene una determinada puntuación, al sumar los puntos ganados en cada una de las preguntas se obtiene una puntuación total que oscila entre 0 y 10 puntos. Si el sujeto tiene entre 0 y 3 puntos se dice que su grado de dependencia es leve, si tiene entre 4 y 6 su grado es moderado y si tiene 7 o más su grado es severo (Tabla I). Conviene recordar que en ocasiones, no es necesario realizar la totalidad del test para conocer el grado de dependencia que el fumador tiene por la nicotina. Se sabe que basta con preguntar al paciente sobre el número de cigarrillos que consume al día, el tiempo que transcurre desde que se levanta hasta que consume el primer cigarrillo y cuál es el cigarrillo que más necesita consumir. Si las respuestas a estas preguntas son que consume 20 o más cigarrillos diarios, que consume el primer cigarrillo en la primera media hora de después de levantarse y que es ese cigarrillo el que más necesita, el fumador debe ser diagnosticado de moderado-severo grado de dependencia por la nicotina ${ }^{14,15}$.

El conocimiento de los resultados del test de Fagerström no sólo nos servirá para conocer el grado de dependencia que el fumador tiene por la nicotina sino que también lo podremos utilizar con otros fines. Se ha demostrado que los fumadores con más alta puntuación en el test necesitan tratamiento

Tabla I

TEST DE FAGERSTRÖM MODIFICADO

\begin{tabular}{|c|c|c|}
\hline Pregunta & Respuesta & Puntuación \\
\hline $\begin{array}{l}\text { ¿Cuánto tiempo tarda en fumar su primer cigarrillo después } \\
\text { de despertarse? }\end{array}$ & $\begin{array}{l}\text { Hasta } 5 \text { minutos } \\
6-30 \text { minutos } \\
31-60 \text { minutos } \\
\text { Más de } 60 \text { minutos }\end{array}$ & $\begin{array}{l}3 \\
2 \\
1 \\
0\end{array}$ \\
\hline $\begin{array}{l}\text { ¿Encuentra dificultad para no fumar en los sitios en que está prohibido } \\
\text { (hospital, cine, biblioteca,...)? }\end{array}$ & $\begin{array}{l}\text { Sí } \\
\text { No }\end{array}$ & $\begin{array}{l}1 \\
0\end{array}$ \\
\hline ¿Qué cigarrillo le desagrada más dejar de fumar? & $\begin{array}{l}\text { El primero } \\
\text { Otros }\end{array}$ & $\begin{array}{l}1 \\
0\end{array}$ \\
\hline ¿Cuántos cigarrillos fuma cada día? & $\begin{array}{l}31 \text { o más cigarrillos/ día } \\
\text { Entre } 21 \text { y } 30 \text { cigarrillos/ día } \\
\text { Entre } 11 \text { y } 20 \text { cigarrillos/ día } \\
\text { Menos de } 11 \text { cigarrillos/ día }\end{array}$ & $\begin{array}{l}3 \\
2 \\
1 \\
0\end{array}$ \\
\hline $\begin{array}{l}\text { ¿Fuma con más frecuencia durante las primera horas después de } \\
\text { levantarse que durante el resto del día? }\end{array}$ & $\begin{array}{l}\text { Sí } \\
\text { No }\end{array}$ & $\begin{array}{l}1 \\
0\end{array}$ \\
\hline $\begin{array}{l}\text { ¿Fuma aunque esté tan enfermo que tenga que guardar cama la mayor } \\
\text { parte del día? }\end{array}$ & $\begin{array}{l}\text { Sí } \\
\text { No }\end{array}$ & $\begin{array}{l}1 \\
0\end{array}$ \\
\hline
\end{tabular}

Puntuaciones:

Menores o iguales a 3 puntos, grado leve de dependencia.

Valores de 4 a 6 puntos implican una dependencia moderada.

Si la puntuación es igual o superior a 7 puntos, grado severo de dependencia. 
farmacológico para dejar de fumar e, incluso se sabe, que dependiendo de cual sea la puntuación se deberá recomendar uno u otro tipo de fármaco para el abandono ${ }^{16-18}$. También se ha encontrado que existe relación entre la puntuación del test y el desarrollo de enfermedades asociadas al consumo del tabaco. Por ejemplo, se ha encontrado que los pacientes fumadores que desarrollan cáncer de pulmón tienen más alto grado de dependencia física por la nicotina, resultados similares se han encontrado para los pacientes que desarrollan EPOC ${ }^{19,20}$.

Vemos cómo el conocimiento de la puntuación del test de Fagerström nos sirve para determinar el grado de dependencia, para indicarnos el mejor tipo de tratamiento farmacológico a realizar y para valorar el riesgo de desarrollar un determinado tipo de patología por parte de ese fumador.

\section{Intentos previos de abandono y motivos de recaídas}

La existencia de intentos fallidos de abandono del tabaco puede ser un indicativo de la dependencia que dicho fumador presenta al mismo por lo que el análisis de los motivos de recaída puede ser de gran importancia para programar un plan terapéutico con mayores probabilidades de éxito.

\section{Medición de niveles de Monóxido de Carbono (CO) en el aire espirado}

La cooximetría es una exploración que nos permite conocer la cantidad de monóxido de carbono que un sujeto tiene en el aire que espira ${ }^{21}$. Esta cantidad esta en relación con su hábito como fumador. Para la medición de este parámetro se utiliza el cooxímetro que es un aparato que mediante una maniobra sencilla e incruenta permite el conocimiento de los niveles de $\mathrm{CO}$ en el aire espirado por el sujeto.

La utilización de la cooximetría en el diagnóstico del tabaquismo es muy recomendable y permitirá al clínico un mejor conocimiento de las características del fumador. En general existe una relación directa entre el número de cigarrillos consumidos y los niveles de $\mathrm{CO}$ en el aire espirado por el fumador. De tal manera que a un mayor número de cigarrillos le corresponde unos más altos niveles de $\mathrm{CO}$ en el aire espirado ${ }^{21}$. No obstante, existen algunos casos en los que a pesar de un escaso consumo de tabaco, se detectan unos altos niveles de $\mathrm{CO}$ o al revés $^{22,23}$. Ello es debido a diferencias en el patrón de consumo de los cigarrillos o, incluso a diferencias de género y sexo. Es decir, es posible que fumadores de un escaso número de cigarrillos diarios tengan altos niveles de $\mathrm{CO}$ en su aire espirado si es que consumen esos cigarrillos dándoles muchas pipadas o inhalando muy profundamente el humo ${ }^{2 f-23}$.
Por ello, el conocimiento de los niveles de $\mathrm{CO}$ nos permitirá diagnosticar con mayor precisión el grado de tabaquismo que un determinado fumador padece. Pero no sólo esto, se sabe que los fumadores con niveles más altos de $\mathrm{CO}$ en su aire espirado desarrollan enfermedades relacionadas con el consumo de tabaco con mayor probabilidad que aquéllos con niveles más bajos ${ }^{20,24}$. Es decir, este parámetro puede ser utilizado como factor de riesgo en un determinado fumador.

Por otro lado, la utilización de la cooximetría tiene indudables ventajas para el fumador. No olvidemos que los fumadores no conocen un parámetro objetivo que les indique el daño que el tabaquismo le está produciendo a su organismo. Esta circunstancia no se da en los hipercolesterolémicos o en los hipertensos, en los que el conocimiento de su cantidad de colesterol o de sus cifras de tensión arterial les ayuda al mejor entendimiento de la mayor o menor gravedad de su enfermedad. Con toda probabilidad cuando los fumadores conozcan sus cifras de $\mathrm{CO}$ en su aire espirado tendrán un mejor conocimiento del daño que el tabaquismo les está causando y ese será un dato que les motivará para dejar de fumar. Igualmente, observar el descenso rápido de la concentración de $\mathrm{CO}$ en la primera visita después de iniciar la abstinencia, respecto del nivel existente cuando aún fumaba, actúa como factor motivador ya que señala la posibilidad de obtener beneficios inmediatos en el proceso de abstinencia.

Además el nivel de $\mathrm{CO}$ en aire espirado puede ser utilizado como marcador real de abstinencia, lo que permite verificar la fiabilidad de la valoración subjetiva de la misma por el paciente y permite al clínico intervenir ante la sospecha de falta de veracidad en la respuesta del paciente. No obstante, antes de asegurar la falta de fiabilidad de la información sobre la abstinencia proporcionada por el paciente, es necesario asegurar que los niveles elevados de $\mathrm{CO}$ no son producidos por otras causas distintas al consumo de tabaco como la contaminación ambiental o la calefacción doméstica.

Luego vemos que la realización de la cooximetría dentro del estudio clínico del fumador no sólo proporciona importante información diagnóstica al médico (grado de tabaquismo, riesgo de desarrollar enfermedad), sino que también puede ayudar al fumador en su proceso de sensibilización y abandono del consumo del tabaco.

Esta técnica con ser aconsejable no debe considerarse totalmente imprescindible y por tanto, supeditar el abordaje terapéutico del paciente fumador a la realización de la misma llevaría consigo, dada la baja disponibilidad actual de realizar cooximetrías en la mayoría de las consultas de Atención Primaria, a una baja cobertura del problema que se está abordando. 
A continuación se expone la técnica para la correcta realización de la cooximetría y cuál es su valoración.

Técnica: el sujeto debe realizar una inspiración profunda y mantener una apnea de 15 segundos, después procederá a una espiración lenta, prolongada y completa. Esperaremos unos segundos hasta que el indicador del cooxímetro se estabilice y marqué el número exacto de ppm de $\mathrm{CO}$ que el sujeto tiene en el aire que espira ${ }^{21}$.

Valoración: niveles de 10 o más ppm de $\mathrm{CO}$ en el aire espirado corresponden a sujetos fumadores. Niveles de 6 a 10 ppm a fumadores esporádicos, y cifras por debajo de 6 ppm a no fumadores ${ }^{21-23}$.

\section{Propuesta de abordaje diagnóstico en el fumador (Fig. 1)}

A continuación exponemos cómo debe abordarse la búsqueda de los datos diagnósticos mínimos en cada fumador. Se establece un protocolo que proporcionará la mayor información en el menor tiempo posible.

Ante todo fumador seguiremos el siguiente protocolo:

1. Número de cigarrillos consumidos al día.

- Menos de 10

-Entre 10 y 20

-De 21 a 30.

-Más de 30

2. Número de años que lleva fumando.

-Menos de 5

- Entre 5 y 10

-Entre 11 y 20

- Más de 20

3. Intentos previos de abandono del tabaco.

- Si existiesen indagar sobre los motivos de recaídas

4. Tiempo que transcurre desde que se levanta hasta que consume el primer cigarrillo del día.

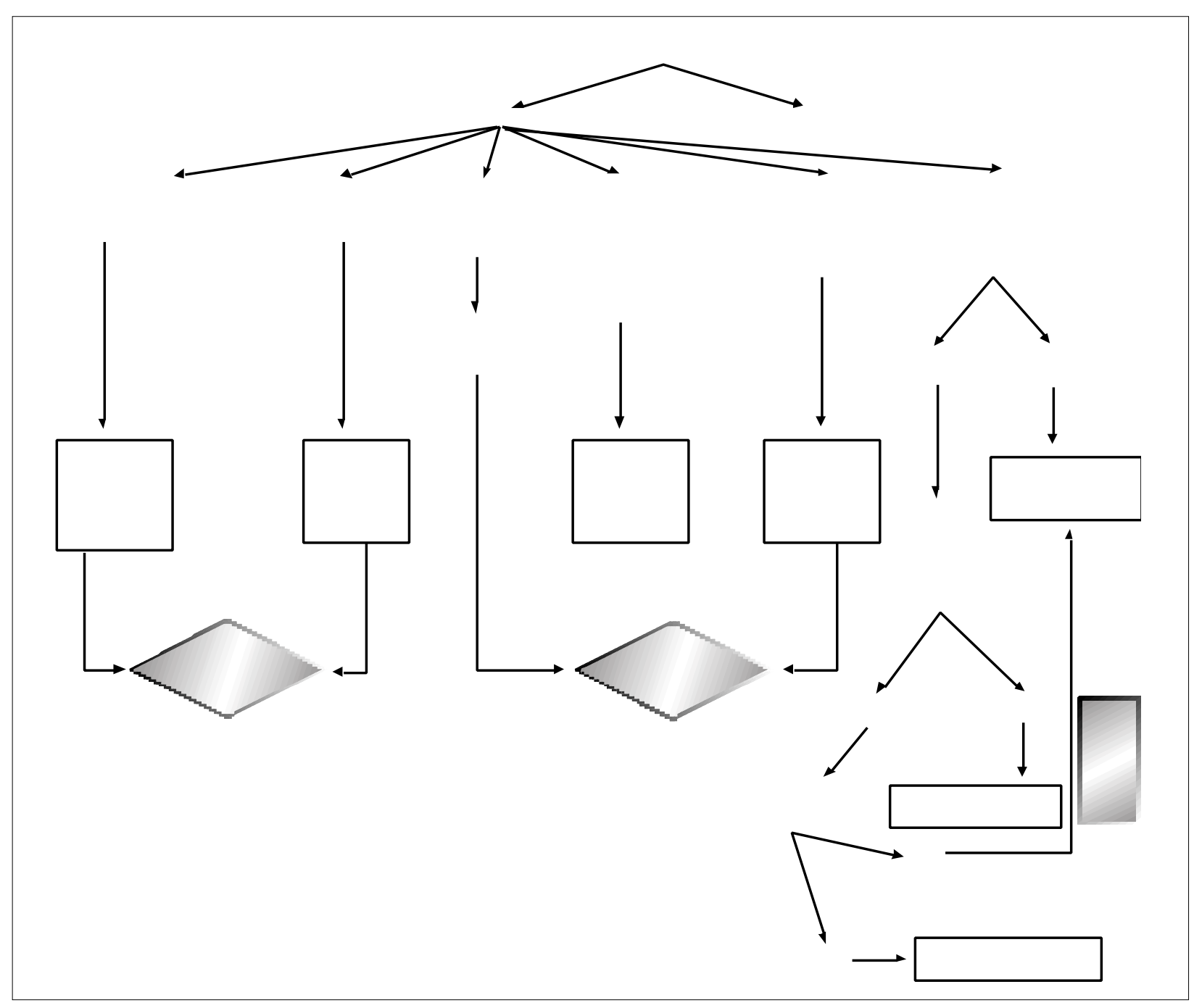

Figura 1

Protocolo de actuación diagnóstica. 
-Menos de 30 minutos.

-Más de 30 minutos.

5. ¿Cuál es el cigarrillo que más necesita?

-El primero del día.

- Cualquier otro.

6. ¿Quiere hacer un serio intento de abandono?

-No. Fase de Precontemplación.

- Sí. En este caso pasaremos a la pregunta 7.

7. ¿Está dispuesto a realizar un serio intento en el próximo mes?

-Sí. Fase de Preparación.

-No. En este caso pasaremos a la pregunta 8.

8. ¿Está dispuesto a realizar un serio intento en los próximos seis meses?

- Sí. Fase de Contemplación.

-No. Fase de Precontemplación.

9. Realización de la cooximetría.

Después de la realización de este protocolo se puede diagnosticar al fumador desde tres puntos de vista: grado de tabaquismo (basándonos en el número de cigarrillos fumados al día, en el número de años de fumador y en los niveles de $\mathrm{CO}$ de su aire espirado), fase de abandono en la que se encuentra y grado de adicción física por la nicotina. Conviene destacar que la cumplimentación completa de este protocolo por parte de un profesional mínimamente entrenado no llevará más de cinco minutos. La gran cantidad de datos que se obtienen con su realización y el escaso consumo de tiempo que conlleva, hacen muy útil y recomendable la puesta en marcha de este protocolo en cualquiera de los niveles asistenciales y por cualquier tipo de profesional sanitario.

\section{Diagnóstico de tabaquismo}

A la luz de los resultados obtenidos en el análisis del conjunto mínimo de datos diagnósticos del fumador podemos, en líneas generales, encuadrar su diagnóstico en cuanto a varios parámetros.

- En cuanto a su grado de tabaquismo.

Los fumadores de 5 paquetes/año o menos y que tienen menos de 15 ppm de $\mathrm{CO}$ en su aire espirado, pueden ser considerados como fumadores con grado leve de tabaquismo.

Los fumadores de más de 5 paquetes/año y menos de 15 paquetes/año y que tienen más de 15 ppm de $\mathrm{CO}$ en su aire espirado, pero menos de 25 deben ser considerados como fumadores con grado moderado de tabaquismo.

Los fumadores de más de 15 paquetes/año y 25 o más ppm de $\mathrm{CO}$ en su aire espirado deben ser considerados como con grado severo de tabaquismo.

Conviene especificar que el parámetro $\mathrm{CO}$ en aire espirado es más potente que el número de paquetes/año para encuadrar al fumador en una de estas categorías ${ }^{7-11,13,20-24}$.

Los datos obtenidos en este apartado nos servirán para establecer un pronóstico y de esta forma ofertar el mejor consejo sanitario al fumador. En principio, todos los fumadores están sometidos a riesgo para su salud por ser consumidores de esta droga, no hay consumo seguro de tabaco, pero aquellos que mayor severidad tienen en su grado de tabaquismo y que, además padecen otros factores de riesgo serían los que mayor riesgo corren de sufrir alguna de las muchas consecuencias que se derivan de su consumo ${ }^{7-11,20-24}$.

-En cuanto a su fase de abandono.

Los fumadores que no se plantean el abandono son fumadores en fase de precontemplación. Aquéllos que lo intentarán en los próximos seis meses están en fase de contemplación, y, por último, los que harán un serio intento de abandono en el próximo mes están en fase de preparación ${ }^{12}$.

El conocimiento de la fase en la que el fumador se encuentra ayudará al sanitario a establecer el más adecuado tratamiento. El principal objetivo del tratamiento en los fumadores en fase de preparación será ayudarles a que definitivamente dejen de fumar, con ello estaremos triplicando sus posibilidades de ser no fumadores a los seis meses después de nuestra intervención, por el contrario el principal objetivo del tratamiento de los fumadores en fase de contemplación o precontemplación será ayudarles a que progresen adecuadamente a fases más avanzadas dentro de su particular proceso de abandono, con ello estaremos doblando sus posibilidades de ser no fumadores a los seis meses después de nuestra intervención ${ }^{12,13,25}$

- En cuanto a su grado de dependencia física por la nicotina.

Los fumadores que consumen más de 20 cigarrillos diarios, fuman su primer cigarrillo del día en la primera media hora de después de levantarse y es ese el que más necesitan, y además refieren intentos previos fallidos por presentar manifestaciones del síndrome de abstinencia a la nicotina son fumadores con moderado-severo grado de dependencia física por la nicotina. Aquéllos que consumen menos de 20 cigarrillos diarios, fuman el primero del día después de los 30 minutos de levantarse y no es ese el que más necesitan, son fumadores con grado leve de dependencia física por la nicotina ${ }^{14,15}$.

Conviene reseñar que la respuesta que el fumador de a la pregunta tiempo que transcurre entre el momento de levantarse y consumo del primer cigarrillo del día es la más potente para diagnosticar el grado de severidad de la dependencia física que por la nicotina tiene el fumador ${ }^{14}$.

Los datos obtenidos en este apartado servirán para aventurar un pronóstico en el fumador. En general, a mayor grado de dependencia mayor posibilidad de desarrollar enfermedad asociada al consumo del tabaco ${ }^{19,20}$. Pero sobre todo para indicar el tratamiento farmacológico más correcto para ese fumador cuando esté dispuesto a realizar un serio intento de abandono ${ }^{16-18}$. 


\section{Abordaje terapéutico}

A continuación, y a modo de orientación, se expone cuál debe ser el abordaje terapéutico de cada fumador de acuerdo a sus características diagnósticas.

- Si es fumador en precontemplación o contemplación lo consideraremos como de bajo o alto riesgo, dependiendo de la presencia o no de otros factores de riesgo y del propio grado de tabaquismo del fumador; es decir, la trascendencia que el consumo de tabaco, de forma independiente o asociada a otras circunstancias, puede tener para su salud. Así: fumadores de 5 o menos paquetes/año, con menos de 15 ppm de $\mathrm{CO}$ en el aire espirado, sin enfermedad relacionada con el consumo del tabaco y sin otros factores de riesgo deben ser considerados como fumadores en precontemplación o contemplación de bajo riesgo. Fumadores de más de 5 paquetes/año, con 15 o más ppm de $\mathrm{CO}$, con enfermedad relacionada con el consumo del tabaco y/o con otros factores de riesgo deben ser considerados como fumadores en precontemplación o contemplación de alto riesgo ${ }^{19-24}$.

- Fumadores en precontemplación o contempla ción de bajo riesgo

Este grupo de fumadores recibirá consejo de abandono de consumo de tabaco/intervención mínima. Este consejo será breve de no más de 3 minutos de duración, a través de él, el profesional sanitario explicará claramente al fumador que dejar de fumar es la medida sanitaria más eficaz que este puede tomar para mantenerse en buena salud, y le alertará sobre como los niveles de $\mathrm{CO}$ en su aire espirado están más elevados que en los no fumadores. Si se acompaña de la entrega de documentación de apoyo por escrito y de apoyo psicológico se englobaría dentro del concepto de intervención mínima en tabaquismo. Grado de evidencia $\mathrm{A}^{26-29}$.

- Fumadores en precontemplación o contempla ción de alto riesgo

En estos fumadores, como en el caso anterior, está especialmente indicada la intervención mínima con todos sus elementos: consejo médico, documentación escrita, apoyo psicológico y seguimiento. Intervención que en función de la fase en la que nos encontremos y características de cada fumador se realizará puntualmente o en cada visita que el fumador realice (intervención mínima sistematizada). El profesional sanitario transmitirá de forma seria y contundente al paciente la necesidad que este tiene de abandonar el consumo de tabaco para mejorar y/o curar su proceso patológico. Igualmente le advertirá de su elevado grado de tabaquismo y de la presencia de otros factores de riesgo que no hacen más que multiplicar sus posibilidades de morbi-mortalidad prematura y de pérdida de calidad de vida. El profesional sanitario se ofrecerá al paciente para ayudarle en la realización de un serio intento de abandono y le citará en un periodo de 4 a 6 semanas para controlar si ha habido algún cambio en su actitud para dejar de fumar. Grado de evidencia $\mathrm{A}^{26-29}$.

-Fumadores en preparación

Este grupo de fumadores recibirá tratamiento para dejar de fumar. El tratamiento podrá ser llevado a cabo en la consulta de medicina de familia o en la Unidad Especializada de Tabaquismo. La consulta de medicina de familia o Atención Primaria, como en el resto de las patologías, debe ser el primer lugar donde el paciente, en este caso el fumador, deba ser atendido o al menos orientado. La profesionalidad del médico de familia y la cobertura y accesibilidad de Atención Primaria hace que este nivel asistencial sea básico y fundamental en el abordaje del tabaquismo, pero existen situaciones que por su complejidad o características acompañantes hacen aconsejable la derivación del fumador a una Unidad Especializada en Tabaquismo cuando consideremos que el simple consejo médico/intervención mínima pueda ser insuficiente, y siempre que exista dicha posibilidad. A continuación exponemos las circunstancias que recomendarán que el sujeto sea derivado a la Unidad $^{30}$.

1. Fumadores que han realizado en el pasado serios intentos de abandono del tabaco que fueron correctamente tratados por un profesional sanitario y que a pesar de ello fracasaron.

2. Fumadores con cardiopatía isquémica de menos de 8 semanas de evolución.

3. Fumadores con arritmias cardiacas o hipertensión arterial no controladas.

4. Fumadores con patología crónica (nefropatía, hepatopatía, cardiopatía, etc.) no controlada.

5. Fumadoras embarazadas o en periodo de lactancia.

6. Fumadores con enfermedades psiquiátricas.

Todos aquellos fumadores que no cumplan estos criterios podrán ser tratados en la consulta del médico de familia o de cualquier especialista del segundo nivel asistencial que integre el tratamiento del tabaquismo dentro de la asistencia global que presta a sus pacientes. El tratamiento de estos fumadores siempre aglutinará dos aspectos. Por un lado, el apoyo psicológico y por otro, el tratamiento farmacológico. Grado de evidencia $\mathrm{A}^{4,13,31-34}$.

El apoyo psicológico será prestado a lo largo de las visitas de seguimiento y tratará de dar consejos al paciente para prepararse para dejar de fumar y para mantenerse sin fumar. Irá acompañado de la entrega de material bibliográfico donde el fumador encontrará por escrito todo tipo de consejos para mantenerse sin fumar. Grado de evidencia $\mathrm{A}^{4,13,31-34}$.

El tratamiento farmacológico debería ser siempre contemplado, en algunas situaciones como tratamiento de base y en otras como refuerzo puntual a la intervención mínima sistematizada, cuando és- 
ta de forma aislada no fuera suficiente para el correcto abordaje de cada fumador, abordaje que como se está viendo ha de ser personalizado y adecuado a las características de cada individuo, y así aumentar las posibilidades de éxito terapéutico.

Fumadores de menos de 5 paquetes/año que consumen su primer cigarrillo después de 30 minutos de levantarse y con menos de 15 ppm de $\mathrm{CO}$ en su aire espirado que corresponda a una dependencia al nicotina baja y un consumo de cigarrillos bajo-moderado. En estos se recomendará la utilización de chicles de nicotina de 2 mg cada 90 ó 120 minutos durante 6 a 8 semanas, reduciendo progresivamente la dosis a partir de la sexta semana o la utilización de parches de nicotina de 24 horas a dosis de $21 \mathrm{mg} /$ día durante 6 semanas y después 14 mg/día durante 2 semanas y después $7 \mathrm{mg}$ /día durante una semana, o parches de nicotina de 16 horas a dosis de $15 \mathrm{mg} /$ día durante 6 semanas y después $10 \mathrm{mg}$ /día durante 2 semanas y después 5 $\mathrm{mg} /$ día durante una semana o bupropión a dosis de $150 \mathrm{mg}$ dos veces al día durante 7 semanas. Grado de evidencia $\mathrm{A}^{4,13,31-34}$.

Fumadores de más de 5 paquetes/año que consumen su primer cigarrillo en los primeros $30 \mathrm{minu}-$ tos de después de levantarse y con más de 15 ppm de $\mathrm{CO}$ en su aire espirado, es decir, con un consumo moderado-alto de cigarrillos y alta dependencia nicotínica. En éstos se recomendará la utilización de chicles de $4 \mathrm{mg}$ de nicotina a dosis de una pieza cada 60-90 minutos durante 12 semanas reduciendo progresivamente a partir de la 10 semana o la utilización de parches de nicotina de 24 horas a do- sis de $21 \mathrm{mg} /$ día durante 6-8 semanas, y después 14 $\mathrm{mg}$ /día durante 2 semanas y después $7 \mathrm{mg} /$ día durante 1 semana, o parches de nicotina de 16 horas a dosis de $25 \mathrm{mg}$ /día durante 6-8 semanas y después $15 \mathrm{mg} /$ día durante 2 semanas y después $10 \mathrm{mg} /$ día durante dos semanas o bupropión $150 \mathrm{mg}$ dos veces al día durante 9 semanas. En algunos de estos pacientes, sobretodo en los que tienen mayor grado de dependencia, estaría recomendada la utilización de spray nasal con nicotina a dosis de $2 \mathrm{mg}$ a $3 \mathrm{mg}$ cada hora mientras que el sujeto este despierto durante un periodo de 3 meses, reduciendo progresivamente la dosis durante tres meses más a razón de un $25 \%$ de reducción mensual. No conviene superar la dosis de más de $5 \mathrm{mg}$ a la hora o más de 40 mg al día ni utilizar esta medicación más allá de seis meses. Otra pauta terapéutica recomendada para estos pacientes es la combinación entre parches y chicles de nicotina a las mismas dosis antes comentadas o la combinación de parches y spray nasal a las dosis recomendadas. Se debe considerar también, el uso combinado de terapia sustitutiva de nicotina y bupropión. Grado de evidencia $\mathrm{A}^{4,13,31-34}$.

CORRESPONDENCIA:

Miguel Torrecilla García

C.S. San Juan

C/ Valencia 32

37005 Salamanca

e-mail: mtorrecillag@papps.org

\section{Bibliografía}

1. Encuesta nacional sobre el hábito de fumar. Ministerio de Sanidad y Consumo. Dirección general de salud pública, 1995.

2. The Smoking Cessation Clinical practice guideline. Panel and staff. The agency for Health Care Policy and research. Smoking Cessation Clinical Practice Guideline. JAMA 1996; 275: 1270-80

3. Banegas Banegas JR, Díez Gañán L, Rodríguez-Artalejo F, González Enríquez J, Graciani A, Villar F. Mortalidad atribuible al tabaquismo en España en 1998. Med Clin (Barc) 2001; 117: 692-4.

4. Fiore MC, Bailey WC, Cohen SJ, Dorfman SF, Goldstein MG, Gritz ER, et al. Treating tobacco use and dependence. Clinical Practice guideline. Rockville, MD:US Department of Health and Human Services. Public Health Service. June 2000.

5. Fiore MC, Jorenby DE, Schnsky AE, Smith SS, Bauer RR, Baker TB. Smoking status as the new vital sign: effect on assessment and intervention in patients who smoke. Mayo Clin Proc 1995; 70: 209-13.

6. Mcbride PE, Plane MB, Underbakke G, Brown RL, Solberg
LI. Smoking screening and management in primary care practices. Arch Fam Med 1997; 6: 165-72.

7. Doll R, Crofton J. Tobacco and health. Br Med Bull, 1996. p. 52 .

8. US Department of Health and Human Services. The health consequences of smoking: Cancer. A report of the Surgeon General. Washington DC. US department of Health and Human Services 1982. DHHS. Publ PHS 82-50179.

9. Burrows B, Knudson RJ, Camilli AE, Stiezer M, Nett LA, Lando H, et al. Quantitative relationship between cigarette smoking and ventilatory function. Am Rev Respir Dis 1988; 137: 286-92.

10. Jarvis MJ. Patterns and predictors of smoking in the general population. En: The Tobacco epidemic. Bolliger CT, Fagerström KO. Prog Respir Res Basel Karger 1997; 28: 151-64.

11. Jarvis MJ. Gender differences in smoking cessation: real o myth? Tobacco control 1994; 3: 324-8.

12. Prochazka J, DiClemente C. Stages and process of selfchange of smoking: towards an integrative model of change. J Clin Psychol 1983; 3: 390-5. 
13. Jiménez Ruiz CA, Solano S, González JM, Ruiz M, Flórez $\mathrm{S}$, Ramos A, et al. Recomendaciones para el tratamiento del tabaquismo. Arch Bronconeumol 1999; 335: 499-506.

14. Fagerström KO, Schneider N. Measuring nicotine dependence: a review of the FTND. J Behav Med 1989; 12: 15982.

15. Heatherton TF, Kozlowsky LT, Frecker RC, Baker T, Whisler W, Fagerström KO. The Fagerström test for nicotine dependence: a revision of the Fagerström tolerance questionnaire. Br J Addict 1991; 86: 1119-27.

16. Tonnesen P. Nicotine replacement and other drugs in smoking cessation. En: Bolliger CT, Fagerström KO. (eds). The Tobacco epidemic. Prog respir res. Basel, Karger 1997; 28: 178-89.

17. Silagy C, Mant D, Fowler G, Lodge M. Meta-analysis on efficacy of nicotine replacement therapies in smoking cessation. Lancet 1994; 343: 139-42.

18. Tonnesen P. Smoking cessation programs. En: Hansen HH (ed): Lung Cancer. Amsterdan, Kluwer. 1994. p. 75-89.

19. Kunze M. Harm reduction: the possible role of nicotine replacement. En: Bolliger CT, Fagerström KO (eds). The tobacco epidemic. Prog respir Res. Basel, Karger 1997; 28: 190-8.

20. Jiménez Ruiz CA, Masa F, Miravitlles M, Gabriel R, Viejo JL, Villasante C, et al. Smoking characteristics: differences in attitudes and dependence between healthy smokers an smokers with COPD. Chest 2001; 119: 1365-70.

21. Jarvis J, Russell MAH. Expired air CO: a simple breath of tobacco smoke intake. BMJ 1980; 281: 484-5.

22. Clark KD, Wardrobe-Wong N, Eliot JJ, Priece T, Lindends $\mathrm{T}$, Larstrom B. Cigarette smoke inhalation and lung damage in smokers volunteers. Eur Respir J 1998; 12: 395-9.

23. Zacny JP, Stitzer ML, Browh FJ, Brown FH, Prawn HN, Lawn HJ, et al. Human cigarette smoking: effect of puff and inhalation parameters on smoke exposure. J Pharmacol Exp Ther 1986; 240: 554-64.
24. Wald NJ, Howard S, Smith PG, Kjeldsen K. Association between atherosclerotic disease and carboxyheamoglobin levels in tobacco smoke. Br Med J 1973. p. 761-5.

25. Prochaska J, Goldstein M. Process of smoking cessation. Implications for clinicians. Clin Chest Med 1991; 12: 727 35.

26. Silagy C. Physician advice for smoking cessation. Oxford: Cochrane Library; N 2. Update software. 2001.

27. Lancaster T, Stead LF. Individual behavioural counselling for smoking cessation. Oxford: Cochrane Library; N 2. Update software. 2001.

28. Rice VH, Stead LF. Nursing interventions for smoking cessation. Oxford: Cochrane Library; N 2. Update software. 2001.

29. Lancaster T, Stead LF. Self-help interventions for smoking cessation. Oxford: Cochrane Library; N 2. Update software. 2002.

30. Jiménez Ruiz CA, Solano Reina S, Barrueco Ferrero M, de Granda Orive JI, Lorza Blasco JJ, Alonso Viteri S, et al. Recomendaciones para la organización y funcionamiento de las unidades especializadas en tabaquismo. Arch Bronconeumol 2001; 37: 382-7.

31. Jiménez Ruiz CA, Ayesta J, Planchuelo MA, Abéngozar R, Torrecilla M, Quintas AM, et al. Recomendaciones de la Sociedad Española de Especialistas en Tabaquismo para el tratamiento farmacologico del tabaquismo. Prev Tab 2001; 3: 78-85.

32. Silagy C, Lancaster T, Stead L, Mant D, Fowler G. Nicotine replacement therapy for smoking cessation. Oxford: Cochrane Library; N 3. Update software. 2001

33. Hughes JR, Stead LF, Lancaster T. Anxiolytics and antidepressants for smoking cessation. Oxford: Cochrane Library; N 2. Update software. 2001

34. US Department of Health and Human Services. Public Health Service. Clinical Practice Guideline: Treating tobacco use and dependence. Washginton. June 2000. 